\title{
Rule Based Rash Driving Alerts System Using IoT and Smart Bike
}

\author{
${ }^{* 1}$ Punit Gupta, ${ }^{2}$ Ankit Mundra, ${ }^{3}$ Aditya Mohan \\ 1,3 Jaypee University of Information Technology, Himachal Pradesh, India \\ ${ }^{2}$ Manipal University, Jaipur, India \\ *Email: punitg07@gmail.com
}

\section{Received: 09 ${ }^{\text {th }}$ July 2018, Accepted: 14 ${ }^{\text {th }}$ August 2018, Published: 31 ${ }^{\text {st }}$ August 2018}

\begin{abstract}
In this paper, we present a smart and safe Bike riding system to provide a safe and an intelligent driving features with accidental, speeding and rash driving alerts. The system is based on the Ethernet based 2nd Generation Intel Galileo Board. This intelligent system will be embedded in the upcoming bikes and motorcycles to prevent speeding and rash driving accidents. The whole idea of the system is to generate an alert to the user and provide caution alert to the user about their driving statistics and warn them as necessary. The system is embedded with various sensors like accelerometers, gyroscope and GPS to make this system an intelligent one. The proposed outcome of the system aims as multiple benefits of preventing accidents, maintaining the ride statistics and getting the directions for the ride. Smart Bike is an IOT based ride system. In today's world, everything is getting automated.
\end{abstract}

Keywords: Internet of Thing (IoT), Power Consumption Smart Devices, Home Automation.

\section{Introduction}

The future of Internet of Things increases the horizons of our vision and also enabling public to access and contribute rich resources about probably everything ever existed in the world. The success of the convergence of the internet of things and the world giving the capability to share experience and personalized insights with the humans also shows great potential for integration with place which need manual labor which can be at some point time taking and inaccurate. Information distribution may be rewarded through inducements, thus transforming the Internet of Things from mere manufacturing commercial applications to an intelligent infrastructure that would reduce human labor and could accomplish task in comparably less time and most accurately also allowing us the trading of enriched information and accelerate business innovation. Being open source and end-user programming will enable people to share to the Internet of Things with data, valued resources and functionalities. IoT has evolved from the convergence of wireless technologies, microelectromechanical systems (MEMS), micro-services and the Internet. The convergence has helped tear down the silo walls between operational technologies (OT) and information technology (IT), allowing unstructured machine-generated data to be analyzed for insights that will drive improvements.

Internet of Things gains its full potential by utilizing the key role playing objects i.e. "Smart" objects which use various sensors and actuators that are able to perceive their context, and via built in networking capabilities they could communicate to each other, access the open source Internet services and interact with the human world. This not only makes the world connected but also robust and comfortable.

Automation is the use of machines or technologies to optimize productivity in the production of goods and delivery of services. The correct incentive for applying automation is to increase productivity, and/or quality beyond that possible with current human labor levels so as to realize economies of scale, and/or realize predictable quality levels. It involves use of various control systems for operating equipment such as machinery, processes in factories, boilers and heat treating ovens, switching in telephone networks, steering and stabilization of ships, aircraft and other applications with minimal or reduced human intervention. Some processes have been completely automated.

The biggest benefit of automation is that it saves labor, however, it is also used to save energy and materials and to improve quality, accuracy and precision. This paper propose an IoT based smart bike ride system that focuses on preventing the increasing road accidents caused due to speedy and rash driving. This Intelligent system will maintain the statistics as well as generate alerts when necessary about the riding style and hence warn the user.

Paper is structured as follows: Section 2 presents existing work from the field of IoT and smart devices for vehicles like car, bike and trains etc. Section 3 discourses the problem statement and proposed model to detect rash driving and alert system. Section 4 describes the simulation setup, scenarios and results as compared to normal and safe driving. Section 5 concludes the work with result outcomes and future work.

\section{Related Work}


A lot of research and development is going in the field of Internet of Things which includes from connecting to devices, collecting data from them, analyzing those large bytes of data and performing the required operation on the data and generates the desired output. Many IOT devices in all the fields including medical and health-care, Home automation, Agriculture etc. have being developed and the same goes for our Smart Petrol Pump solution system. Some of the related IOT devices are discussed below.

\section{1. eCall}

eCall [2] is a service in Europe which serves as an example of an authority undertaking which aims to increase telematics of vehicles. By the time of mentioning, the grand European coalition had set its target to install the eCall model by 2015[10]. This entire system is fixed inside a vehicle and is attached to the air-bag system to sense a collision in real-time It's provided with a user-operated button to ask for assistance for a co-passenger with body issues or to notify the concerned authorities of some other vehicle. It has been equipped with a Global Positioning Service incoming-acceptor for finding out the vehicle and its anticipated route before the mis-happening took place. eCall is triggered by itself when the embedded sensors or trigger-attachments find out of a damaging collision.

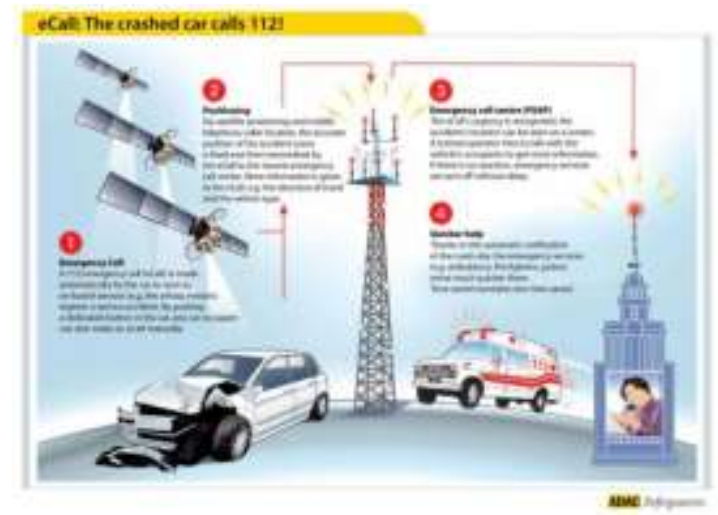

Figure 1. Ecall System

After this service is executed for the first time, the contact sequence of the European Union (which is 112), sets-up a cellphone link to the apt quick response unit quarters (or public safety assuring centers), sending the detail of the happening (or Minimum Set of Data -> M.S.D.) to the savior teams (also incorporating the time of occurrence), the tothe-point location of the affected vehicle and the direction in which the vehicle headed. An eCall can be initiated by thy-self on pressing a button given on the vehicle, eg. by an individual who had a look at the scene.

\section{Contran 245 [9]}

In Brazil, a legislation was presented which demanded all fresh vehicles manufactured or brought to Brazil to be installed with GPRS enabled locating systems for bringing down car thefts. This idea is on its way to fruition.

This bill or act has the ability to transform Brazil into one of the biggest telematic market in the universe, including car tracing and other services, containing simple tracing as needed by the act to features including fleet care-taking and setup and emotion response monitoring for insurance issues.

\section{MIROAD}

Here Johnson et al [1] places vehicle driving styles into average, hostile and ultra-hostile. Like the above cases, sensors like accelerometers, gyroscopes, cameras and magnetometers) on an Iphone were used with the data generated into a singular classification program based on the DTW (Dynamic Time Warping) algorithm. That system "spoke" i.e. gave an audible output for alert generation i.e. if the threshold values were exceeded.

In the U.S., a significant amount of insurance companies have begun implementing service consumption-based insurance to reduce the costs for their customers and also to advocate secure driving.

In Italy gains of service consumption-based insurance has resulted into a new law. Private companies like Octo Telematics provide recommendations for insurance and other tasks relating to the automotive industry by accumulating vehicle location and accident data along with driver behavior info. To support its pay-as-you-drive policies.

Some developments have taken place in utilization of mobile devices (cell-phones) for calculation of various parameters and alert provision. As an example, an app. On the Android platform made by Chirgupta et al [7] which provides quality charts of that specific driver. They had incorporated an accelerometer, a global positioning service sensor and the in-built camera device for capturing the feed. Ranges for accelerating securely were mentioned in the code and values breaking beyond their extremes (thresholds) marked the happening of the associated event.

\section{Proposed Model}

In the proposed model we introduce a smart and safe riding intelligent system that provides the driver, along with the concerned authorities (parents etc.) with safe and intelligent driving features including that of accident alerts, rash driving and speeding alerts. The system is based upon on an Inter Galileo Gen 2 (Quark Processor) which acts as the transmission-brain of the 
whole system. The system is connected to the network via Internet and a database is maintained consisting of the whole ride details, the speed statistics and alerts if any. This system helps the one concerned person in relationship to bike driver and the concerned authority to study and stop rash drivers. The system consists of an Atmega328 based Arduino Uno. The sensors used are GPS module, gyroscope and Accelerometer which details us upon the velocity as well as the tilt of the motorcycle. With the help of the gyroscope we are able to determine the tilt angle and the rotation of the motorbike and with the help of accelerometer, the velocity of the motorbike is calculated.

Our system responds to the speed alert when a motorbike crosses a threshold speed which can be determined by the accelerometer. It can also detect whether the person is doing rash driving with the help of sudden rotational change readings in the gyroscope. The gyroscope can determine the rotational reading of the bike and its tilt angle and thus can help to detect the rash driving occurrence. The proposed system focuses on the guidelines set by the local official authorities which include maximum speed, minimum speed and sudden acceleration which are altogether combined with the measured tilt angle to generate a tested and calibrated parameter which single-handedly generates an alert for rash driving which is sent to the user and stored in the database. The authorities which can utilize the alert and stored data include parents, government officials in-case of any lawsuit filing, the data can act as a conduit for showing the statistics of the moment of event occurrence, thereby helping legal undertakings proceed at an accelerated rate. Also, the intelligent system is also able to determine the accidents through sudden change in an orientation of the motorbike and will let you know if you are over speeding in an area where speed limit is restricted.

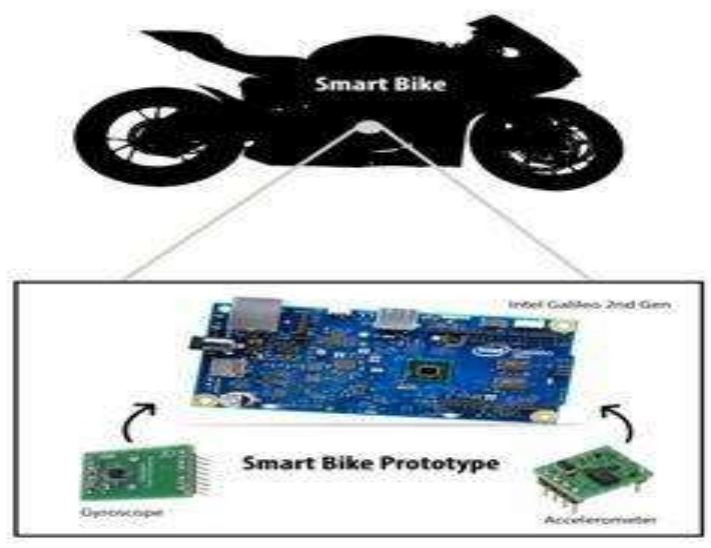

Figure.2. Physical Layout cum Component Set-up of the Proposed Model

The entire process can be executed in parallel- in the first case, it can be used for direct data retrieval and alert generation. In the second case, location tracking can be enabled using the GPS available on the user's end. In this case guidelines issued by the local governing authorities shall influence the threshold values for alert generation.

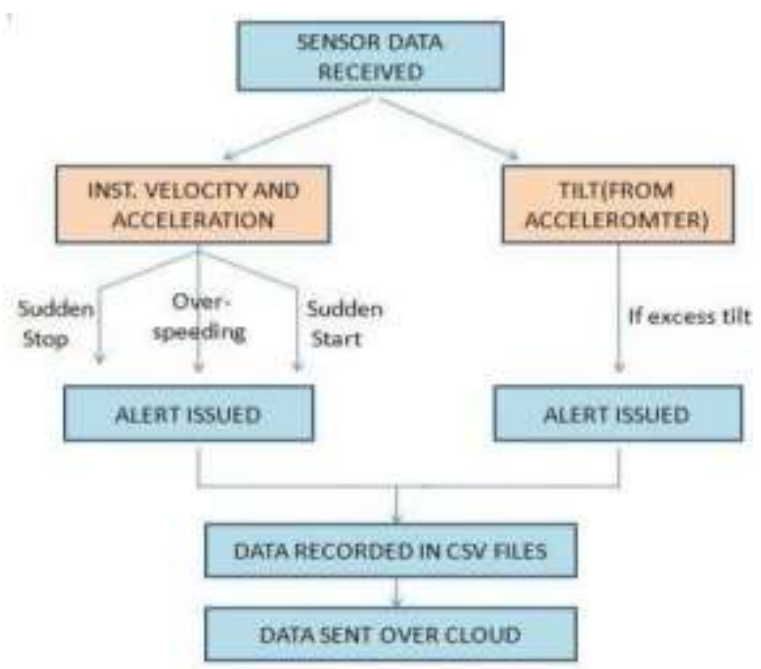

Figure. 3 Pictorial Representation of the Working of the System.

The software part consists of a database server which helps to maintain the statistics of the ride details. The user can also set the GPS details for the ride using his smartphone by connecting it via the Bluetooth. The system uses the Internet server to send the details of the location of the bike in case of accidents or rash driving. Real-time drive-statistics (with various alert parameters recorded) are sent via the Internet for complete data logging and future reference.

The hardware part consists of a GY-61 3-Axis accelerometer and the gyroscope sensor. The sensor used here is MPU6050.The MPU-6050 devices combine a 3-axis gyroscope and a 3-axis accelerometer on the same silicon die, together with an on board Digital Motion. Figure 4 and 5 shows the working of the individual sensors and how they are used for measuring the above-mentioned parameters.

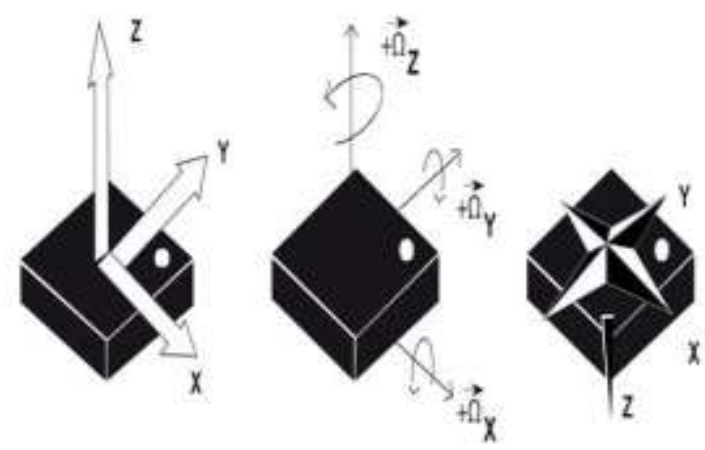

Figure 4. Dynamics of the Gyroscope for Angle Measurement 


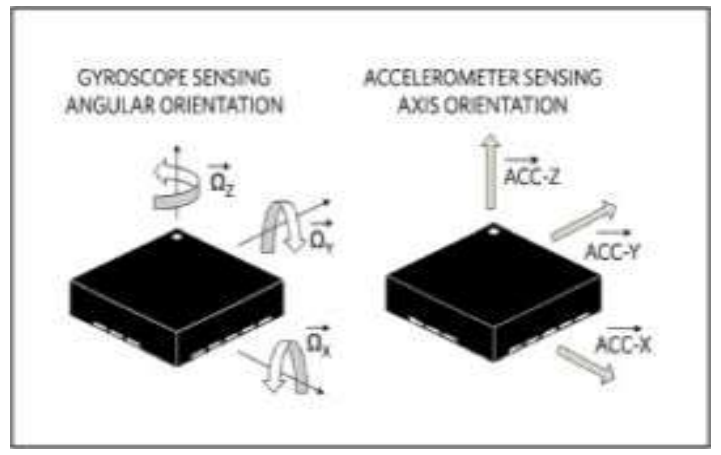

Figure 5. Axial Positioning of the Sensors

\section{Proposed Rule Set}

We have framed out and deduced 4 conditions for categorizing any form of driving as rash driving, which include crossing a defined speed limit, sudden stop of a speeding vehicle, sudden acceleration of a stationary or almost stationary vehicle, and excess tilt of the bike on that specific instantaneous velocity at that moment of time which may result in skidding and Sudden stop may surprise the neighbor vehicles resulting in crash.

Set of rules and conditions are as follows:

\section{- Crossing the Speed Limit}

Here we have defined a specific speed limit of $70 \mathrm{~km} / \mathrm{hr}$ above which if the vehicle goes, then an alert of "speed limit crossed" is generated and stored in the database. The data generated is for a specific time instant and if continued over a long period of time (more than 5-10 continuous iterations), would indicate intentional or self-imposed rash driving.

\section{- Sudden Stop}

If a moving vehicle suddenly stops(i.e. acceleration reduces significantly when its final velocity is highly reduced), then it indicates that either the vehicle was suddenly stopped via application of heavy brakes or either it as bumped into another solid, heavier object, either ways, both being the result of rash driving.

\section{- $\quad$ Sudden Acceleration}

If a person accelerates the bike all of a sudden then it indicates that either the vehicle was being mishandled (i.e. being driven under some influence etc.) or it was bumped from behind by another object- both indicating towards rash driving by any of the associated parties.

\section{- Excess Tilt of the Bike}

If at any specific speed, if the vehicle tilts(about its centre of mass) excessively for a prolonged time period then it indicates that the person is sure to get his vehicle flipped, his knees raptured, or bump into any other object or vehicle having a probability of causing heavy damage to life and property.

\section{- Authority Rules from Map}

The road layout taken for simulating the vehicle driving was of Downtown Manhattan (Lower Manhattan, New York, NY, USA), as shown on Figure 6. The simulation was performed in broad daylight conditions with the presence of mild traffic. The starting point of the road trip was from the 259 Adams Street near Brooklyn Bridge $\left(40^{\circ} 41^{\prime} 45.6^{\prime \prime} \mathrm{N}\right.$ $\left.73^{\circ} 59^{\prime} 19.3^{\prime \prime} \mathrm{W}\right)$ to 6th Ave, Brooklyn, NY, USA $\left(40^{\circ} 38^{\prime} 14.5^{\prime \prime} \mathrm{N} 74^{\circ} 00^{\prime} 53.7^{\prime \prime} \mathrm{W}\right)$ with an estimated ride time of 11 minutes. Total length of the road covered for making the observations was 6.8 miles. The road comprised of an estimated amount of 14 turns (including both sharp and mild turns).



Figure 6. Map Downtown Manhattan (Lower Manhattan, New York, NY, USA).

\section{- Specifications of Hardware Used}

Microcontroller: Inter Galileo Gen 2 (Quark Processor).

Sensors: GY-61 3-Axis accelerometer, MPU-6050 gyroscope

\section{Experimental Results}

The proposed IoT based Smart Bike System has been simulated with sensors been attached on bike with GPS connectivity. The testing is been done over road map over Downtown Manhattan(Lower Manhattan, New York, NY, USA) with all the rules of maximum speed at specific point of road from Google maps as shown in figure 6.

Figure 7 shows comparison of acceleration change in driving of normal driver and rash driving as shown with blue and red color in graph. Figure shows a drastic fluctuation in speed as compared to smooth change in velocity in normal driver. Figure 8 shows alert generated due to sudden change in acceleration as shown in green and red shows the acceleration change due to rash driving as compared to a dataset of normal driver. 


\section{Conclusion}

From experimental result section, it is clear that proposed intelligent system is the best in its kind for providing a better alerts and can detect more type of mis-driving as compared to normal driving skills The main idea of this system is to minimize the road accidents which are increasing day by day by alerting and warning the driver of their ride styles and providing them the best security necessary and also send the alerts to the parents and one concerned person about the driving behavior of the driver. State authority can use this data for grabbing and finding the driver with these unfit driving skills and can be used to study the driving behavior or a specific area.

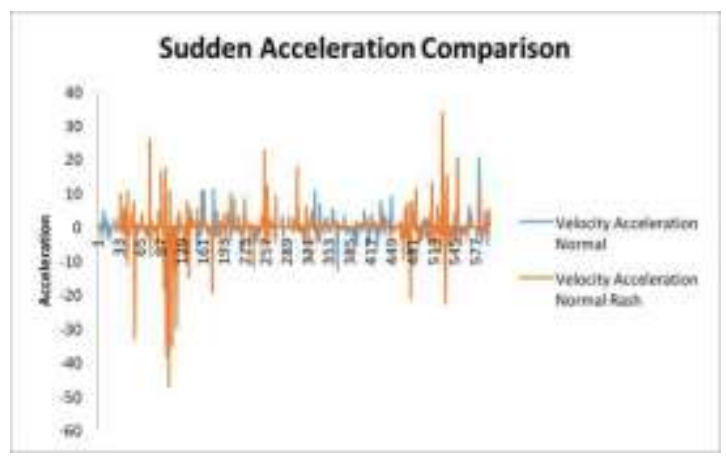

Figure. 7 Sudden of Acceleration Change

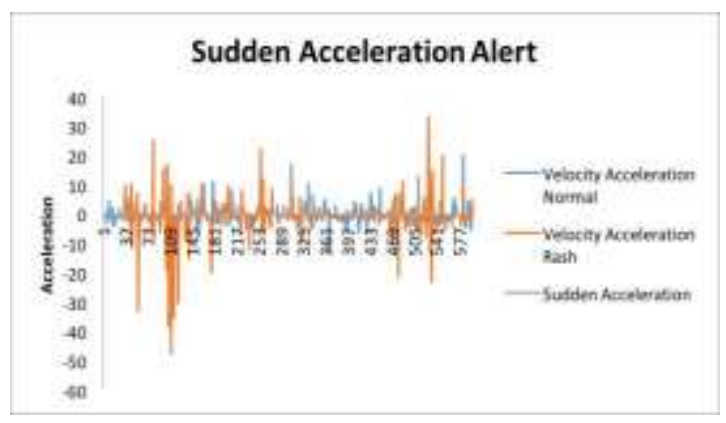

Figure. 8 Sudden Acceleration Alert

\section{References}

1. Johnson DA, Trivedi MM. Driving style recognition using a smartphone as a sensor platform. InIntelligent Transportation Systems (ITSC), 2011 14th International IEEE Conference on 2011 Oct 5 (pp. 1609-1615). IEEE.

2. Pinart C, Calvo JC, Nicholson L, Villaverde JA. ECall-compliant early crash notification service for portable and nomadic devices. InVehicular Technology Conference, 2009. VTC Spring 2009. IEEE 69th 2009 Apr 26 (pp. 1-5). IEEE.

3. Schietekat JM, Booysen MJ. Detection of reckless driving in the Sub-Saharan informal public transportation system using acceleration-sensing telematics. InEUROCON, 2013 IEEE 2013 Jul 1 (pp. 597-601). IEEE.

4. Shubhada Vital Poojary, Rashmi, Sudheer Shetty,"Humps and Pothole Detection and Alerting System for Safe Journey", International Research Journal of Engineering and Technology (IRJET) Volume: 03 Issue: 05 | May-2016

5. Chu HL, Raman V, Shen J, Choudhury R, Kansal A, Bahl V. In-vehicle driver detection using mobile phone sensors. InACM MobiSys 2011 Apr 20.

6. Kalra N, Chugh G, Bansal D. Analyzing driving and road events via smartphone. International Journal of Computer Applications. 2014 Jul;98(12):5-9.

7. Chen K, Lu M, Fan X, Wei M, Wu J. Road condition monitoring using on-board three-axis accelerometer and GPS sensor. InCommunications and Networking in China (CHINACOM), 2011 6th International ICST Conference on 2011 Aug 17 (pp. 1032-1037). IEEE.

8. Zhang Y, Lin WC, Chin YK. A pattern- recognition approach for driving skill characterization. IEEE Transactions on Intelligent Transportation Systems. 2010 Dec;11(4):905-16.

9. Jalali S. M2M solutions-Design challenges and considerations. InIntelligent Computational Systems (RAICS), 2013 IEEE Recent Advances in 2013 Dec 19 (pp. 210-214). IEEE. 